\title{
Identification of clinical risk factors of atrial fibrillation in congestive heart failure
}

\author{
Ryszard Targoński, ${ }^{1,}$, Janusz Sadowski ${ }^{2}$, Jerzy Romaszko ${ }^{3}$, Leszek Cichowski ${ }^{2}$ \\ ${ }^{1}$ University of Warmia and Mazury in Olsztyn, Faculty of Medicine, Olsztyn, Poland \\ ${ }^{2}$ Cardiology and Internal Diseases Department, Urban Hospital in Olsztyn, Olsztyn, Poland \\ ${ }^{3}$ NZOZ Pantamed Sp. z o.o., Olsztyn, Poland
}

\begin{abstract}
Background: Factors associated with the development of atrial fibrillation $(A F)$ in general population have been described, but it is still unknown whether the same risk factors apply to heart failure (HF) patients. The aim of this study was to identify clinical factors related to various forms of $A F$ in $H F$ patients.

Methods: The clinical and echocardiographic characteristics were assessed in 155 HF patients: 50 with sinus rhythm, 52 with non-permanent $A F$, and 53 with permanent $A F$.

Results: Multivariate logistic regression analysis showed that the increase in the NYHA class was an independent risk factor for both forms of $A F$. The occurrence of permanent $A F$ in comparison to sinus rhythm group was independently associated with hs-C-reactive protein (CRP) elevation above $1 \mathrm{mg} / \mathrm{dL}$ (OR 1.87, 95\% CI 1.05-3.35), left atrial dimension above $4 \mathrm{~cm}$ (OR 3.78, 95\% CI 1.29-11.06) and tricuspid maximal pressure gradient elevation above $35 \mathrm{~mm} \mathrm{Hg}$ (OR 5.01, 95\% CI 1.38-18.27). The presence of coronary disease was independently associated with less frequent occurrence of permanent $A F$ in comparison to sinus rhythm group (OR 0.21, 95\% CI 0.06-0.67).

Conclusions: More advanced congestive HF was associated with presence of both types of AF. Non-ischemic etiology of HF and elevated CRP are independently associated with permanent AF compared to sinus rhythm. Left ventricular diastolic dysfunction indicators (increased tricuspid maximal pressure gradient and left artial dimension) are independently associated with permanent AF. (Cardiol J 2013; 20, 4: 364-369)
\end{abstract}

Key words: heart failure, atrial fibrillation, echocardiography, C-reactive protein, coronary disease

\section{Introduction}

The advent of modern treatments in cardiology and the resulting survival benefit in industrialized countries have considerably increased the size of the population of patients suffering from congestive heart failure (CHF), estimated at over 15 million in the European Union [1].
Coronary artery disease (CAD), hypertension and tachyarrhythmia, usually in the form of atrial fibrillation (AF), are believed to be the most common causative factors of myocardial dysfunction [2-4]. The development of AF in CHF patients further exacerbates the signs and symptoms of $\mathrm{HF}$, increases the risk of thromboembolic events and adversely affects the long-term prognosis [3].

Address for correspondence: Ryszard Targoński, MD, PhD, Cardiology and Internal Diseases Department, Urban Hospital in Olsztyn, ul. Niepodleglosci 44, 10-045 Olsztyn, Poland, tel/fax: +48 8952722 35, e-mail: rtarg@op.pl 
While factors associated with the development of $\mathrm{AF}$ in the general population of patients have been identified [5], it remains unclear whether the same risk factors apply to the population of patients suffering from $\mathrm{CHF}$. The known causes of $\mathrm{AF}$ in patients with $\mathrm{CHF}$ include rapidly progressive conditions, such as hyperthyroidism, electrolyte imbalance, acute myocardial ischemia, alcohol abuse and heart surgery [3]. Maisel and Stevenson [6] reported an increased incidence of AF with increasing severity of $\mathrm{CHF}$, as assessed using the New York Heart Association (NYHA) functional classification. It has not been established whether there is an independent association between $\mathrm{AF}$ and the increasing severity of $\mathrm{CHF}$ assessed using the NYHA classification or between $\mathrm{AF}$ and $\mathrm{CHF}$ assessed by echocardiographic parameters. Studies in patients without HF describe an association of $\mathrm{AF}$ with markers of inflammation $[7,8]$. In the case of $\mathrm{AF}$ in patients with $\mathrm{CHF}$ this association has not yet been elucidated [9].

Identification of factors associated with the development and persistence of cardiac arrhythmias is of important prognostic significance and may play a role in therapy if these factors can be modified.

The aim of the study was to identify factors associated with various forms of $\mathrm{AF}$ in the population of patients with CHF.

\section{Methods}

This was a cross-sectional study investigating a population of patients with CHF. The study was approved by the local ethics committee. All the patients met the European Society of Cardiology (ESC) diagnostic criteria of $\mathrm{CHF}$ [3]. Patients with acute (up to 3 months duration) and chronic inflammatory diseases, hyperthyroidism, hemodynamically significant valvular disease, NYHA class IV CHF, or cancer were excluded from the study. The study population consisted of patients referred to the Cardiology Clinic by 41 general practitioners over a period of 15 months. Consecutive patients were assigned to one of the following 3 groups: the sinus rhythm (SR) group, the non-permanent $\mathrm{AF}$ (npAF) group (3 patients with paroxysmal and 49 with persistent $\mathrm{AF}$ ) or to the permanent $\mathrm{AF}$ (pAF) group. The ESC classification of AF was followed [5].

We assessed the baseline demographic and clinical characteristics of the study patients. The severity of $\mathrm{CHF}$ was established in accordance with the NYHA functional classification. The diagnosis of hypertension was based on the history or measurements of blood pressure values in accordance with the ESH/ESC guidelines [10]. The diagnosis of $\mathrm{CAD}$ was established in patients with a history of ST-elevation myocardial infarction or based on an angiographically confirmed significant narrowing of an epicardial coronary artery. The diagnoses of diabetes mellitus and metabolic syndrome were established in accordance with the International Diabetes Federation criteria [11]. Conduction abnormalities in the form of bundle branch blocks or atrioventricular block were diagnosed by ECG and/ /or based on a history of a pacemaker implantation. Laboratory and echocardiographic assessments were ordered during the first study visit. The patients underwent transthoracic echocardiography performed with GE Vingmed System FIVE using the standard 2.5-5.0 MHz probe. The methods of the study have been reported previously [9].

The results are presented as frequency values or calculated mean values for the analyzed groups of patients.

Preliminary statistical analysis of the study parameters allowed us to determine whether the percentage of patients within a particular class of a given parameter is independent of the group of patients classified on the basis of the presence and type of $\mathrm{AF}$ and whether the mean values of a given parameter in individual groups are similar or significantly different.

The distribution of hs-C-reactive protein (CRP) levels was not normal, hence the natural logarithm of the hs-CRP levels (ln CRP) was used for further analyses.

The mean values in the groups were compared using parametric tests (the t-Student or Cochrane-Cox tests) or the non-parametric U Mann-Whitney test.

Some continuous variables were transformed into dichotomized variables using their upper limits of normal as threshold values. Patients with left ventricular ejection fraction (LVEF) values below $45 \%$ made up Class 1 , while the remaining patients made up Class 2. Patients with the maximum pressure gradient across the tricuspid valve (tMxPG) values below $35 \mathrm{~mm} \mathrm{Hg}$ made up Class 1, while the remaining patients made up Class 2 . The remaining variables were categorized as follows: NYHA functional classes were categorized in 3 classes; hs-CRP levels in 3 classes $(\leq 1.00 \mathrm{mg} / \mathrm{L}, 1.01-$ $-3.00 \mathrm{mg} / \mathrm{L}$ and $>3.00 \mathrm{mg} / \mathrm{L}$ ) [12, 13]; left atrial (LA) dimension in 3 classes $(\leq 4 \mathrm{~cm}, 4.01-5.00 \mathrm{~cm}$ and $>5.00 \mathrm{~cm}$ ) [9].

Univariate logistic regression analysis was used to determine which of the analyzed variables were significantly associated with the particular 
Table 1. Patients characteristics.

\begin{tabular}{|c|c|c|c|c|c|c|}
\hline \multirow[t]{3}{*}{ Analyzed parameters } & \multicolumn{5}{|c|}{ Patient groups by type of arrhythmia } & \multirow[t]{3}{*}{$P^{* *}$} \\
\hline & \multirow{2}{*}{$\begin{array}{c}\text { SR } \\
N=53\end{array}$} & \multicolumn{2}{|c|}{ Non-permanent AF } & \multicolumn{2}{|c|}{ Permanent AF } & \\
\hline & & $\mathbf{N}=\mathbf{5 2}$ & $\mathbf{P}^{*}$ & $\mathbf{N}=\mathbf{5 0}$ & $\mathbf{P}^{*}$ & \\
\hline Age & $72.2 \pm 9.2$ & $76.2 \pm 10.0$ & 0.038 & $74.5 \pm 9.9$ & NS & NS \\
\hline Male & $37(69.8 \%)$ & $34(65.4 \%)$ & NS & $30(60.0 \%)$ & NS & NS \\
\hline Hypertension & $40(75.5 \%)$ & $39(75.0 \%)$ & NS & $38(76.0 \%)$ & NS & NS \\
\hline Diabetes mellitus & $48(90.6 \%)$ & $22(42.3 \%)$ & $<0.001$ & $11(22.0 \%)$ & $<0.001$ & 0.033 \\
\hline Coronary artery disease & $34(64.1 \%)$ & $30(57.7 \%)$ & NS & $17(34.0 \%)$ & 0.003 & 0.017 \\
\hline Dilated cardiomyopathy & $3(5.7 \%)$ & $3(5.8 \%)$ & NS & $6(12.0 \%)$ & NS & NS \\
\hline Mitral insufficiency & $4(7.5 \%)$ & $7(13.5 \%)$ & NS & $15(30.0 \%)$ & 0.005 & NS \\
\hline Systolic heart failure & $32(60.4 \%)$ & $37(71.2 \%)$ & NS & $36(72.0 \%)$ & NS & NS \\
\hline NYHA class III & $9(17.0 \%)$ & $22(42.3 \%)$ & 0.006 & $28(56.0 \%)$ & $<0.001$ & NS \\
\hline Peripheral edema & $20(37.7 \%)$ & $26(50.0 \%)$ & NS & $29(58.0 \%)$ & 0.045 & NS \\
\hline Conduction abnormalities & $24(50.9 \%)$ & $19(36.5 \%)$ & NS & $22(44.0 \%)$ & NS & NS \\
\hline BMI $\left[\mathrm{kg} / \mathrm{m}^{2}\right]$ & $28.6 \pm 5.1$ & $29.4 \pm 5.0$ & NS & $29.4 \pm 6.5$ & NS & NS \\
\hline Metabolic syndrome & $24(45.3 \%)$ & $23(44.2 \%)$ & NS & $15(30.0 \%)$ & NS & NS \\
\hline ACEI & $50(94.3 \%)$ & $50(96.2 \%)$ & NS & $44(88.0 \%)$ & NS & 0.014 \\
\hline ARB & 0 & 0 & & 6 & & \\
\hline Beta-blocker & $44(83.0 \%)$ & $46(88.5 \%)$ & NS & $45(90.0 \%)$ & NS & NS \\
\hline Statin & $46(86.8 \%)$ & $45(86.5 \%)$ & NS & $28(56.0 \%)$ & 0.001 & 0.001 \\
\hline Spironolactone & $34(64.2 \%)$ & $38(73.1 \%)$ & NS & $40(80.0 \%)$ & NS & NS \\
\hline Diuretics & $35(66.0 \%)$ & $36(69.2 \%)$ & NS & $44(88.0 \%)$ & 0.010 & 0.022 \\
\hline Aspirin & 45 (84.9\%) & $44(84.6 \%)$ & NS & $26(52.0 \%)$ & 0.001 & 0.001 \\
\hline Acenocoumarol & $4(7.5 \%)$ & $24(46.2 \%)$ & $<0.001$ & $37(74.0 \%)$ & $<0.001$ & 0.005 \\
\hline
\end{tabular}

${ }^{*}$ vs. SR, ${ }^{* *}$ non-permanent AF vs. permanent AF; SR — sinus rhythm; AF — atrial fibrillation; BMI — body mass index; ACEI — angiotensin-converting enzyme inhibitors; ARB — angiotensin receptor blockers

types of AF. The variables for which the significance level was below 0.10 were used as the baseline variables for multivariate logistic regression analysis. An optimal subset of variables was identified. The odds ratio was estimated from the following formula: $\mathrm{OR}=\mathrm{OR}_{\mathrm{AxB}}=\mathrm{S}(\mathrm{X}=\mathrm{A}) / \mathrm{S}(\mathrm{X}=\mathrm{B})=\mathrm{P}(\mathrm{Y}$ $\left.=\left.1\right|_{\mathrm{X}=\mathrm{A}}\right) / \mathrm{P}\left(\mathrm{Y}=\left.1\right|_{\mathrm{X}=\mathrm{B}}\right)$, for $\mathrm{A}>\mathrm{B}$, where $\mathrm{A}$ and $\mathrm{B}$ referred to classes of the independent factor $\mathrm{X}$.

A significance level of 0.05 was adopted to verify the statistical hypotheses. The statistical analysis was performed using Statistica 6.0 PL.

\section{Results}

Baseline characteristics of the 3 study groups and the results of the statistical tests to verify the significant differences between the groups are presented in Table 1 . Although the patients in the npAF group were older than the patients in the remaining 2 groups, the difference was statistically significant only vs. the SR group $(p=0.038)$. CAD was significantly more common in the SR and the npAF groups than in the $\mathrm{pAF}$ group ( $\mathrm{p}=0.03$ and $\mathrm{p}=0.017$, respectively). The highest percentage of patients with diabetes mellitus was identified in the SR group $(p<0.001$ for both comparisons). This percentage decreased with increasing severity of AF. The prevalence of haemodynamically significant mitral insufficiency and peripheral edema was significantly higher in the pAF group vs. the SR group ( $\mathrm{p}=0.005$ and $\mathrm{p}=0.045$, respectively). The prevalence of NYHA class III HF was significantly higher in the npAF and $\mathrm{pAF}$ groups than in the SR group $(\mathrm{p}=0.06$ and $\mathrm{p}<0.001$, respectively). Table 1 presents also the details of the pharmacological treatment in the study groups. The differences in pharmacotherapy resulted from the underlying cause and severity of $\mathrm{CHF}$ and from the presence of AF. The exception was the treatment with angiotensin-converting enzyme inhibitors, which was less common in the $\mathrm{npAF}$ group than in the $\mathrm{pAF}$ group as a result of the more frequent use of angiotensin receptor blockers in the pAF group (6 patients).

The results of laboratory and echocardiographic assessments in the study groups are summari- 
Table 2. Comparison of laboratory and echocardiographic parameters between the study groups.

\begin{tabular}{|c|c|c|c|c|c|c|}
\hline \multirow[t]{2}{*}{ Analyzed parameters } & \multirow{2}{*}{$\begin{array}{c}\text { SR } \\
N=53\end{array}$} & \multicolumn{2}{|c|}{ Non-permanent AF } & \multicolumn{2}{|c|}{ Permanent AF } & \multirow[t]{2}{*}{$\mathbf{P} * *$} \\
\hline & & $N=52$ & $\mathbf{P}^{*}$ & $N=50$ & $\mathbf{P}^{*}$ & \\
\hline hs-CRP [mg/L] & $4.27 \pm 11.2$ & $8.81 \pm 27.3$ & & $8.84 \pm 11.6$ & & \\
\hline In hs-CRP & $0.67 \pm 1.11$ & $1.13 \pm 1.27$ & 0.052 & $1.56 \pm 1.20$ & $<0.001$ & NS \\
\hline Fibrinogen [mg/dL] & $361.4 \pm 79.6$ & $355.5 \pm 65.6$ & NS & $398.0 \pm 87.2$ & 0.021 & 0.007 \\
\hline Hemoglobin [g/dL] & $14.0 \pm 1.5$ & $13.4 \pm 1.7$ & NS & $13.9 \pm 1.6$ & NS & NS \\
\hline $\operatorname{MCV}\left[\mu \mathrm{m}^{3}\right]$ & $91.4 \pm 4.0$ & $91.6 \pm 5.6$ & NS & $91.2 \pm 6.1$ & NS & NS \\
\hline Platelets $\left[\times 10^{3} / \mathrm{mL}\right]$ & $194.5 \pm 55.8$ & $182.3 \pm 52.1$ & NS & $186.1 \pm 63.5$ & NS & NS \\
\hline WBC $\left(\times 10^{3} / \mathrm{mL}\right)$ & $7.6 \pm 2.0$ & $7.4 \pm 2.0$ & NS & $7.3 \pm 2.1$ & NS & NS \\
\hline Serum creatinine [mg/dL] & $0.99 \pm 0.39$ & $1.10 \pm 0.57$ & NS & $1.12 \pm 0.30$ & 0.010 & NS \\
\hline $\mathrm{Na}^{+}[\mathrm{mmol} / \mathrm{L}]$ & $139.5 \pm 2.8$ & $140.7 \pm 3.4$ & 0.025 & $140.3 \pm 3.1$ & NS & NS \\
\hline $\mathrm{K}^{+}[\mathrm{mmol} / \mathrm{L}]$ & $4.33 \pm 0.41$ & $4.48 \pm 0.40$ & NS & $4.39 \pm 0.47$ & NS & NS \\
\hline Glucose [mg/dL] & $106.1 \pm 25.1$ & $109.4 \pm 33.9$ & NS & $103.7 \pm 32.8$ & NS & NS \\
\hline Total cholesterol [mg/dL] & $177.6 \pm 45.2$ & $158.5 \pm 34.7$ & 0.036 & $163.9 \pm 47.3$ & NS & NS \\
\hline Triglycerides [mg/dL] & $137.6 \pm 82.3$ & $119.7 \pm 70.1$ & NS & $121.8 \pm 70.1$ & NS & NS \\
\hline HDL-cholesterol [mg/dL] & $54.2 \pm 14.7$ & $50.7 \pm 16.0$ & NS & $51.9 \pm 16.5$ & NS & NS \\
\hline LDL-cholesterol [mg/dL] & $95.9 \pm 43.0$ & $83.8 \pm 32.6$ & NS & $87.7 \pm 40.0$ & NS & NS \\
\hline LA [cm] & $4.40 \pm 0.58$ & $4.56 \pm 0.81$ & NS & $5.20 \pm 0.77$ & $<0.001$ & $<0.001$ \\
\hline LV [cm] & $5.90 \pm 1.03$ & $5.86 \pm 0.98$ & NS & $5.85 \pm 1.08$ & NS & NS \\
\hline LVEF [\%] & $45.7 \pm 13.8$ & $42.1 \pm 12.7$ & NS & $43.7 \pm 14.1$ & NS & NS \\
\hline $\mathrm{RV}[\mathrm{cm}]$ & $2.62 \pm 0.40$ & $2.49 \pm 0.52$ & NS & $2.80 \pm 0.58$ & NS & 0.004 \\
\hline $\mathrm{tMxPG}[\mathrm{mm} \mathrm{Hg}]$ & $16.9 \pm 17.7$ & $18.6 \pm 15.0$ & NS & $36.2 \pm 17.4$ & $<0.001$ & $<0.001$ \\
\hline
\end{tabular}

${ }^{*}$ vs. SR, ** non-permanent AF vs. permanent AF; SR - sinus rhythm; AF - atrial fibrillation; CRP - C-reactive protein; MCV - mean corpuscular volume; LA — left atrium; LV — left ventricle; RV — right ventricle; LVEF — left ventricular ejection fraction; tMxPG — tricuspid maximal pressure gradient

zed in Table 2. The mean hs-CRP levels (ln CRP) were higher in the npAF and pAF groups than in the SR group ( $\mathrm{p}=0.052$ and $\mathrm{p}<0.001$, respectively). The pAF group was also characterized by a significantly higher fibrinogen levels compared to the other 2 groups $(\mathrm{p}=0.021$ and $\mathrm{p}=0.007$, respectively) and by a significantly higher serum creatinine levels compared to the SR group $(p=0.010)$. Sodium levels were significantly higher $(\mathrm{p}=0.025)$ and total cholesterol significantly lower $(\mathrm{p}=0.036)$ in the npAF group, compared to the SR group. There were no significant differences in LVEF between the npAF or pAF groups and the SR group. LA dimension was significantly higher in the pAF group $(\mathrm{p}<0.001$ and $\mathrm{p}<0.001$, respectively) as was tMxPG $(\mathrm{p}<0.001$ and $\mathrm{p}<0.001$, respectively). Right ventricular (RV) dimension was higher in the pAF group compared to the npAF group $(\mathrm{p}=0.004)$.

The parameters showing a statistically significant association with the presence of arrhythmia on univariate logistic regression analysis were: diabetes mellitus, CAD, NYHA class of CHF, the levels of hs-CRP, fibrinogen, serum creatinine and sodium, and LA dimension, RV dimension and tMxPG. The parameters for which the significance level did not exceed 0.10 were the baseline parameters for multivariate logistic regression analysis, which allowed us to identify an optimal subset of factors of which the most important ones may be treated as variables independently associated with a particular type of AF.

The final results of the multivariate logistic regression analysis for the above types of $\mathrm{AF}$ are summarized in Table 3.

The optimal subset of parameters describing the likelihood of npAF included: NYHA class of CHF, hs-CRP levels and LA dimension, treated as the classified variables. The increase in the severity of CHF, as assessed by the NYHA functional class, was the only variable independently associated with npAF.

In addition, the optimal subset of independent variables described by the likelihood of pAF also included: confirmed CAD and elevated tMxPG. The multivariate logistic regression analysis revealed that elevated hs-CRP, NYHA class increase, LA enlargement and $\mathrm{tMxPG}$ increase above $35 \mathrm{~mm} \mathrm{Hg}$ were independently associated with pAF, whereas $\mathrm{CAD}$ was independently associated with reduced odds of pAF after multivariable adjustment. 
Table 3. Multivariable analysis; optimal subsets of independent variables.

\begin{tabular}{|c|c|c|c|c|c|c|}
\hline \multirow{2}{*}{$\begin{array}{l}\text { Independent } \\
\text { variables X } \\
\text { Factors (number } \\
\text { of classes) }\end{array}$} & \multirow{2}{*}{$\begin{array}{l}\text { Regression } \\
\text { coefficients }\end{array}$} & \multirow[t]{2}{*}{$\mathbf{P}$} & \multicolumn{2}{|c|}{$\mathrm{OR}^{* *}$} & \multicolumn{2}{|c|}{$95 \% \mathrm{Cl}^{* * *}$} \\
\hline & & & $\begin{array}{l}\text { One class } \\
\text { change }\end{array}$ & $\begin{array}{l}\text { Range } \\
\text { changes }\end{array}$ & $\begin{array}{l}\text { One class } \\
\text { change }\end{array}$ & $\begin{array}{l}\text { Range } \\
\text { changes }\end{array}$ \\
\hline \multicolumn{7}{|c|}{ III $A-Y^{*}: A F(1-$ non-permanent $A F ; 0-$ sinus rhythm) } \\
\hline Intercept & -2.701 & & & & & \\
\hline NYHA (3 classes) & 1.243 & 0.001 & 3.47 & 12.01 & $1.70-7.08$ & $2.88-50.15$ \\
\hline hs-CRP (3 classes) & 0.123 & NS & 1.14 & 1.45 & $0.74-1.72$ & $0.41-5.12$ \\
\hline LA (3 classes) & 0.297 & NS & 1.35 & 1.81 & $0.68-2.66$ & $0.46-7.09$ \\
\hline \multicolumn{7}{|c|}{ III $B-Y^{*}: A F(1-$ permanent AF; $0-$ sinus rhythm) } \\
\hline Intercept & -7.747 & & & & & \\
\hline CAD & -1.582 & 0.008 & 0.21 & & $0.06-0.67$ & \\
\hline NYHA (3 classes) & 1.508 & 0.004 & 4.52 & 20.40 & $1.61-12.70$ & $2.57-161.38$ \\
\hline hs-CRP (3 classes) & 0.755 & 0.033 & 1.87 & 6.56 & $1.05-3.35$ & $1.14-37.68$ \\
\hline LA (3 classes) & 1.484 & 0.014 & 3.78 & 14.28 & $1.29-11.06$ & $1.67-122.36$ \\
\hline tMxPG & 1.719 & 0.013 & 5.01 & & $1.38-18.27$ & \\
\hline \multicolumn{7}{|c|}{ III $C-Y^{*}: A F(1-$ permanent AF; 0 - non-permanent AF) } \\
\hline Intercept & -2.52 & & & & & \\
\hline CAD & -0.865 & 0.061 & 0.42 & & $0.17-1.05$ & \\
\hline hs-CRP (3 classes) & 0.491 & NS & 1.63 & 4.37 & $0.97-2.76$ & $0.91-21.08$ \\
\hline LA (3 classes) & 1.135 & 0.003 & 2.9 & 8.41 & $1.42-5.92$ & $2.02-35.03$ \\
\hline tMxPG & 1.411 & 0.004 & 4.1 & & 1.54-10.89 & \\
\hline
\end{tabular}

*Dichotomized variable $Y$ describing the type of atrial fibrillation $(1$ - success; 0 - failure); ** Odds ratio (OR) for the change in the value of the independent variable; ***95\% confidence interval for OR; AF — atrial fibrillation; CAD — coronary artery disease; tMxPG — tricuspid maximal pressure gradient; $L A$ - left atrium

$\mathrm{OR}=\mathrm{OR}_{\mathrm{AxB}}=\mathrm{P}\left(\mathrm{Y}=\left.1\right|_{\mathrm{x}=\mathrm{A}}\right) / \mathrm{P}\left(\mathrm{Y}=\left.1\right|_{\mathrm{X}=\mathrm{B}}\right), \mathrm{A}>\mathrm{B}->$ number of classes of variable $\mathrm{X}$

Factors significantly and independently associated with $\mathrm{pAF}$, when the failure was npAF, turned out to be: increased LA dimension and elevated tMxPG above $35 \mathrm{~mm} \mathrm{Hg}$.

\section{Discussion}

Although the CHF patients in both $\mathrm{AF}$ groups were older than those in the SR group, the difference was not independently associated with $\mathrm{AF}$. A similar finding of the higher prevalence of $\mathrm{AF}$ among older patients was reported in the EuroHeart Failure Survey [14]. We did not identify any association between the arrhythmia and hypertension, or increasing obesity, as assessed by body mass index, which are factors associated with $\mathrm{AF}$ in the general population [5]. The analysis of a large European population of patients with $\mathrm{HF}$ conducted by Rivero-Ayerza et al. [14] also demonstrated no significant differences in the prevalence of obesity and hypertension between patients with $\mathrm{AF}$ and patients with SR, which confirms our findings obtained in a relatively small randomly selected group of patients.
Diabetes mellitus and CAD are considered to be the factors that are associated with the development of $\mathrm{AF}$ in general population of patients [5]. In our group of patients with HF the prevalence of diabetes mellitus and CAD were the highest in the SR group and decreased with the severity of the arrhythmia. In a multivariate logistic regression analysis, the diagnosis of CAD was associated with about a 5 -fold decrease in odds ratio for the development of pAF vs. the SR group. In this context, it is notable that Lee et al. [15], in the Framingham population with CHF, showed a significantly higher prevalence of $\mathrm{AF}$ in patients in whom CHF resulted from non-coronary causes. Similarly, Rivero-Ayerza et al. [14], in a population of patients with CHF, observed that $\mathrm{CAD}$ and diabetes mellitus were significantly less prevalent in patients with $\mathrm{CHF}$ and $\mathrm{AF}$ compared to the population of CHF patients with SR. Our study confirmed these findings and showed an independent association between CAD and the less common occurrence of pAF.

Univariate logistic regression analysis shows that both $\mathrm{npAF}$ and $\mathrm{pAF}$ are significantly associated 
with elevated hs-CRP levels. In multivariate analysis, this association has only been confirmed for pAF. The higher was its value, the higher was the likelihood of developing AF. Chung et al. [8] were the first who reported the association between $\mathrm{AF}$ and increasing hs-CRP levels, relative to the severity of $\mathrm{AF}$ in patients without $\mathrm{HF}$. In the case of patients with $\mathrm{CHF}$, this association remains unclear. Shah et al. [16] demonstrated an association between serum hs-CRP and increasing LV end-diastolic pressure. Smith et al. [17] showed that the occurrence of AF was independently associated with elevated MR-proANP and hs-CRP. These findings suggest that hs-CRP elevation may be associated with increased vascular wall tension, which may explain its independent association with LA enlargement and hypertension in the group of patients with $\mathrm{CHF}$ [9].

We also observed that the prevalence of $\mathrm{AF}$ increases with the severity of $\mathrm{CHF}$ as assessed using the NYHA functional classification, which may result from the increasing predisposition to developing $\mathrm{AF}$ with increasing filling pressures in the atria and their enlargement $[5,6]$. We confirmed this association and showed its independent nature. An increase of one NYHA class resulted in an almost 3.5-fold increase in odds ratio for developing $\mathrm{npAF}$ and a 4.5 -fold increase in odds ratio for developing $\mathrm{pAF}$.

The LA enlargement and the elevated $\mathrm{tMxPG}$ values we observed were independently associated with pAF. The higher were these parameters, the higher was the likelihood of developing this arrhythmia. An association between both of these parameters has been shown in the course of CHF. Berkowitz et al. [18] observed that the severity of pulmonary hypertension increased with the severity of LV diastolic dysfunction. It also has been shown that LA volume was depended on the severity of LV diastolic dysfunction [19]. These findings suggest that the disordered LV diastole significantly affects both these parameters. LVEF values did not affect the occurrence of $\mathrm{AF}$ in the study population. In a similar population of patients, Rivero-Ayerza et al. [14] also showed no differences in LVEF between patients with AF and patients without this arrhythmia. Straburzyńska-Migaj et al. [20] observed that in patients with systolic HF and SR, a considerably impaired LV diastolic function is independently associated with pulmonary hypertension. LA enlargement and elevated tMxPG may therefore indirectly indicate an association between $\mathrm{LV}$ diastolic dysfunction with pAF in the course of CHF both in patients with preserved and in patients with impaired LV systolic function.

\section{Conclusions}

More advanced $\mathrm{CHF}$ was associated with presence of both types of AF. Non-ischemic etiology of $\mathrm{HF}$ and elevated CRP are independently associated with permanent AF compared to SR. LV diastolic dysfunction indicators (increased tMxPG and LA dimension) are independently associated with permanent $\mathrm{AF}$.

\section{Conflict of interest: none declared}

\section{References}

1. Senni M, Tribouilloy CM, Rodeheffer RJ et al. Congestive heart failure in the community: Trends in incidence and survival in a 10-year period. Arch Intern Med, 1999; 159: 29-34.

2. Fox KF, Cowie MR, Wood DA et al. Coronary artery disease as the cause of incident heart failure in the population. Eur Heart J, 2001; 22: 228-236.

3. Dickstein K, Cohen-Solal A, Filippatos G et al. ESC Guidelines for the diagnosis and treatment of acute and chronic heart failure 2008: the Task Force for the Diagnosis and Treatment of Acute and Chronic Heart Failure 2008 of the European Society of Cardiology. Eur Heart J, 2008; 29: 2388-2442.

4. Elliott P, Andersson B, Arbustini E et al. Classification of the cardiomyopathies: a position statement from the European Society Of Cardiology Working Group on Myocardial and Pericardial Diseases. Eur Heart J, 2008; 29: $270-276$.

5. Camm AJ, Kirchhof P, Lip GY et al. Guidelines for the management of atrial fibrillation: the Task Force for the Management of Atrial Fibrillation of the European Society of Cardiology (ESC). Eur Heart J, 2010; 31: 2369-2429.

6. Maisel WH, Stevenson LW. Atrial fibrillation in heart failure: epidemiology, pathophysiology, and rationale for therapy. Am J Cardiol, 2003; 91: 2D-8D.

7. Celebi OO, Celebi S, Canbay A, Ergun G, Aydogdu S, Diker E. The effect of sinus rhythm restoration on high-sensitivity C-reactive protein levels and their association with long-term atrial fibrillation recurrence after electrical cardioversion. Cardiology, 2011; 118: 168-174.

8. Chung MK, Martin DO, Sprecher D et al. C-reactive protein elevation in patients with atrial arrhythmias: inflammatory mechanisms and persistence of atrial fibrillation. Circulation, 2001; 104: 2886-2891.

9. Targoński R, Salczyńska D, Sadowski J, Cichowski L. Relationship between inflammatory markers and clinical patterns of atrial fibrillation in patients with congestive heart failure. Kardiol Pol, 2008; 66: 729-736;

10. Mancia G, De BG, Dominiczak A et al. 2007 ESH-ESC Practice Guidelines for the Management of Arterial Hypertension: ESH-ESC Task Force on the Management of Arterial Hypertension. J Hypertens, 2007; 25: 1751-1762.

11. International Diabetes Federation. The IDF consensus worldwide definition of the metabolic syndrome. http://www.idf.org/webdata/docs/Metac_syndrome def.pdf (14 April 2005).

12. Zarauza J, Rodríguez Lera MJ, Fariñas Alvarez C et al. Relationship between C-reactive protein level and early recurrence of atrial fibrillation after electrical cardioversion. Rev Esp Cardiol, 2006; 59: 125-129.

13. Casaclang-Verzosa G, Barnes ME, Blume G et al. C-reactive protein, left atrial volume, and atrial fibrillation: a prospective study in high-risk elderly. Echocardiography, 2010; 27: 394-399.

14. Rivero-Ayerza M, Scholte Op Reimer W, Lenzen M et al. New-onset atrial fibrillation is an independent predictor of in-hospital mortality in hospitalized heart failure patients: results of the EuroHeart Failure Survey. Eur Heart J, 2008; 29: 1618-1624.

15. Lee DS, Gona P, Vasan RS et al. Relation of disease pathogenesis and risk factors to heart failure with preserved or reduced ejection fraction: insights from the Framingham Heart Study of the National Heart, Lung and Blood Institute. Circulation, 2009; 119: 3070-3077.

16. Shah SJ, Marcus GM, Gerber IL et al. High-sensitivity C-reactive protein and parameters of left ventricular dysfunction. J Card Fail, 2006; 12: 61-65.

17. Smith JG, Newton-Cheh C, Almgren P et al. Assessment of conventional cardiovascular risk factors and multiple biomarkers for the prediction of incident heart failure and atrial fibrillation. J Am Coll Cardiol, 2010; 56: 1712-1719.

18. Berkowitz R, Alhaj E, Manchikalapudi RB, Satya K, Dadfarmay S, Zakir R. Determinants of right ventricular failure in patients admitted with acute left heart failure. Congest Heart Fail, 2010; 16: 243-248.

19. Tsang TS, Barnes ME, Gersh BJ, Bailey KR, Seward JB. Left atrial volume as a morphologic expression of left ventricular diastolic dysfunction and relation to cardiovascular risk burden. Am J Cardiol, 2002; 90: 1284-1289.

20. Straburzyńska-Migaj E, Szyszka A, Trojnarska O, Cieśliński A. Restrictive filling pattern predicts pulmonary hypertension and is associated with increased BNP levels and impaired exercise capacity in patients with heart failure. Kardiol Pol, 2007; 65: 1049-1055. 